\title{
Formation of neutrophil extracellular traps in mitochondrial DNA-deficient cells
}

\author{
Yutaka Takishita, ${ }^{1}$ Hiroyuki Yasuda, ${ }^{1}$ Mio Shimizu, ${ }^{1}$ Akane Matsuo, ${ }^{1}$ Akihiro Morita, ${ }^{1}$ Tomonari Tsutsumi, ${ }^{1}$ \\ Masahiko Tsuchiya ${ }^{2}$ and Eisuke F. Sato ${ }^{1, *}$
}

\begin{abstract}
${ }^{1}$ Department of Biochemistry, Faculty of Pharmaceutical Sciences, Suzuka University of Medical Science, 3500-3 Minamitamagaki, Suzuka-city, Mie 513-8670, Japan ${ }^{2}$ Department of Anesthesiology, Osaka City University Medical School, 1-5-7 Asahi-machi, Abeno, Osaka 545-8586, Japan
\end{abstract}

(Received 26 August, 2019; Accepted 5 September, 2019; Published online 12 November, 2019)

\begin{abstract}
Neutrophil extracellular trap (NET) formation plays an important role in inflammatory diseases. Although it is known that NET formation occurs via NADPH oxidase (NOX)-dependent and NOXindependent pathways, the detailed mechanism remains unknown. Therefore, in this study, we aimed to elucidate the role of mitochondria in NOX-dependent and NOX-independent NET formation. We generated mitochondrial DNA-deficient cells $\left(\rho^{0}\right.$ cells) by treating HL-60 cells with dideoxycytidine and differentiated them to neutrophil-like cells. These neutrophil-like $\rho^{0}$ cells showed markedly reduced NOX-independent NET formation but not NOXdependent NET formation. However, NET-associated intracellular histone citrullination was not inhibited in $\rho^{0}$ cells. Furthermore, cells membrane disruption in NOX-dependent NET formation occurred in a Myeloperoxidase (MPO) and mixed lineage kinase domain like pseudokinase (MLKL)-dependent manner; however, cell membrane disruption in NOX-independent NET formation partially occurred in an MLKL-dependent manner. These results highlight the importance of mitochondria in NOX-independent NET formation.
\end{abstract}

Key Words: neutrophil extracellular trap, NETosis, mitochondria, peptidylarginine deiminase 4

$\mathrm{N}$ eutrophils are the first immune cells to respond to pathogen invasion and play a critical role in the subsequent immune response. They are the most abundant leukocytes in circulation and are first recruited to the infected sites. Here, the neutrophils are activated, and these activated neutrophils destroy the pathogens via generation of reactive oxygen species (ROS), phagocytosis, and formation of neutrophil extracellular traps (NETs). ${ }^{(1)}$ NETs are composed of DNA fibers, histones, and antimicrobial proteins such as myeloperoxidase (MPO) and neutrophil elastase released by neutrophils to capture and kill bacteria. ${ }^{(2)}$ NET formation has been reported in cancer, diabetes, and autoimmune diseases such as systemic lupus erythematosus and rheumatoid arthritis. ${ }^{(3-4)}$ Moreover, NETs bind to platelets, thereby causing vascular damage and arteriosclerosis. ${ }^{(5)}$ Although regulation of NET formation is considered to contribute to the prevention of exacerbation of the pathological condition, the detailed mechanisms underlying NET formation are not fully elucidated.

NET formation occurs NADPH oxidase (NOX)-dependent or NOX-independent mechanisms. NOX inhibitors were shown to inhibit both ROS generation and NET formation. ${ }^{(6)}$ Moreover, neutrophils isolated from patients with chronic granulomatous disease (CGD), which is characterized by impaired NOX activity, failed to generate ROS and did not show NET formation. ${ }^{(7)}$ Thus, the NOX-generated ROS play an important role in NET formation. However, it has been reported that neutrophils can extrude NETs via NOX-independent mechanisms in response to stimulants such as calcium ionophores (e.g., A23187) ${ }^{(8,9)}$ Furthermore, it was reported that the calcium ionophore-induced NOX-independent NET formation occurred via small conductance calcium-activated potassium channel protein 3 (SK3) and mitochondrial ROS. ${ }^{(10)}$

Mitochondria are multifunctional organelles that, produce ATP and regulate cell proliferation, differentiation, and oxidative signaling pathways. Therefore, mitochondrial dysfunction and oxidative stress in and around mitochondria have been implicated in pathogenetic mechanisms, including inflammation and autoimmune reactions. Using mitochondrial DNA (mtDNA)-deficient macrophages, we recently reported that mitochondria contribute to intracellular oxidative stress, which is responsible for stimulation of lipopolysaccharide-induced mitogen-activated protein kinase (MAPK) signaling to enhance cytokine release. ${ }^{(11,12)}$ However, the detailed relation between mitochondria and NOX-independent NET formation remains unclear. Therefore, in this study, we generated mtDNA-dificient $\left(\rho^{0}\right)$ cells created using dideoxycytidine (ddC) treatment in HL-60 cells and investigated the role of mitochondria in NOX-dependent and -independent NET formation.

\section{Materials and Methods}

Murine neutrophil isolation. Ten-week-old male C57BL/6 mice (SLC, Hamamatsu, Shizuoka, Japan) and gp91 ${ }^{\text {phox }}$ knockout (KO) mice (Jackson Laboratories, Bar Harbor, ME) were used in the experiments. These mice were bred and housed individually in a specific pathogen-free barrier facility at $23^{\circ} \mathrm{C}$ with 12 -h light/dark cycles. They were provided standard laboratory chow (CE-2, Oriental Yeast Co., Tokyo, Japan) and drinking water. This study was approved by the institutional animal ethics committee and was performed in strict accordance with the recommendations of the Guide for the Care and Use of Laboratory Animals of the Suzuka University of Medical Science (approval number: 21). For the isolation of infiltrating neutrophils, C57BL/6 and gp $91^{\text {phox }}$ $\mathrm{KO}$ mice were intraperitoneally administered $2 \mathrm{ml}$ of $2.98 \%$ thioglycollate (Becton, Dickinson and Company, Franklin Lakes, $\mathrm{NJ}$ ) in PBS. At $4 \mathrm{~h}$ after the administration, neutrophils infiltrating the peritoneal cavity were collected using PBS. The isolated neutrophils were washed three times with PBS and then used for experiments.

Cell culture and mtDNA-deficient cell ( $\rho^{0}$ cell) generation. The human promyelocytic leukemia cell line, HL-60 (RCB3683, RIKEN BioResource Center, Ibaraki, Japan) was cultured in RPMI 1640 medium (Nacalai, Kyoto, Japan) containing 10\% (v/v) heat-inactivated fetal bovine serum and antibiotics in $5 \% \mathrm{CO}_{2}$ humidified air at $37^{\circ} \mathrm{C}$. $\rho^{0}$ cells were produced by culturing HL-60 cells with $1 \mu \mathrm{M}$ (final concentration) ddC for 7 days in the presence of uridine and pyruvic acid. HL-60 and $\rho^{0}$ cells were differ-

*To whom correspondence should be addressed.

E-mail: efsato@suzuka-u.ac.jp 
entiated into neutrophil-like cells by treatment with $1.25 \%$ dimethyl sulfoxide (DMSO) or $1 \mu \mathrm{M}$ all-trans retinoic acid (ATRA) for 3 days, as described previously. ${ }^{(13)}$

Quantification of extracellular DNA. Neutrophil-like HL60 and $\rho^{0}$ cells pretreated with or without 4-aminobenzoic acid hydrazide (ABAH; MPO inhibitor) for $3 \mathrm{~h}$, MitoTEMPO (mitochondrial ROS scavenger) for $30 \mathrm{~min}$, or necrosulfonamide [NSA; mixed lineage kinase domain like psedokinase (MLKL) inhibitor] for $30 \mathrm{~min}$ were seeded at $1 \times 10^{6} \mathrm{cell} / \mathrm{ml}$ in 96 -well plates. These cells were treated with $10 \mu \mathrm{M}$ A23187 or $10 \mathrm{nM}$ phorbol myristate acetate (PMA) for $3 \mathrm{~h}$, whereas the murine neutrophils were treated with $10 \mu \mathrm{M}$ A23187 or $1 \mu \mathrm{M}$ PMA for $3 \mathrm{~h}$. Then, all the cells were treated with $20 \mathrm{U} / \mathrm{ml}$ micrococcal nuclease (New England Biolabs Japan, Tokyo, Japan) for $20 \mathrm{~min}$ at $37^{\circ} \mathrm{C}$. The DNA containing supernatants were collected after centrifugation at $200 \times g$ for $8 \mathrm{~min}$ at $4^{\circ} \mathrm{C}$. Extracellular DNA was transferred to a microwell plate, stained using SYTOX green, and quantified using SpectraMax $^{\circledR}$ (485 nm excitation; $525 \mathrm{~nm}$ emission; Molecular Devices Japan, Tokyo, Japan), and expressed as fold change with respect to the control.

Quantification of NET-associated cell death (NETosis).

NETosis was quantified using a SYTOX green assay. Briefly, neutrophil-like HL-60 and $\rho^{0}$ cells pre-treated with or without ABAH, MitoTEMPO, or NSA were seeded at $1 \times 10^{6} \mathrm{cell} / \mathrm{ml}$ in 96 well plates and treated with $10 \mu \mathrm{M}$ A23187 or $10 \mathrm{nM}$ PMA. The murine neutrophils were treated with $10 \mu \mathrm{M}$ A23187 or $25 \mathrm{nM}$ PMA. The rate of NETosis was quantified hourly using SpectraMax $^{\circledR}$ (485 $\mathrm{nm}$ excitation, $525 \mathrm{~nm}$ emission) in the presence of SYTOX green. To calculate the relation of NETosis, fluorescence of the cells with $1 \%(\mathrm{v} / \mathrm{v})$ Tritone X-100 was considered as $100 \%$ DNA, and NETosis at each time was showed at the $\%$ of total DNA.

NET visualization. To observe NET formation, neutrophils and neutrophil-like HL-60 and $\rho^{0}$ cells were seeded at $2 \times 10^{4}$ cells in flexiPERM ${ }^{\circledR}$ chamber inserts (OLYMPUS, Tokyo Japan) (pore size; $1.8 \mathrm{~cm}^{2}$ ) on a grass slide and incubated with $10 \mu \mathrm{M} \mathrm{A} 23187$ or $10 \mathrm{nM}$ PMA. Then, the cells were incubated in SYTOX green for $5 \mathrm{~min}$. Subsequent changes in fluorescence were observed using confocal microscopy.

Western blotting. Cell samples were suspended in RIPA buffer and sonicated. Aliquots $(15-30 \mu \mathrm{g})$ of the samples were loaded on SDS/PAGE gels. The electrophoresed samples were transferred on to PVDF or protein nitrocellulose membranes via a semi-dry transfer. The membranes were blocked by incubation in $5 \%$ non-fat milk in Tris-buffered saline with Tween 20 . Then, the membranes were incubated with the following primary antibodies at $4{ }^{\circ} \mathrm{C}$ overnight: anti-cytochrome c $(1: 1,000 ; 6 \mathrm{H} 2 . \mathrm{B} 4556432$; BD Bioscience), anti-mitochondria complex I-V (1:1,000; Total OXPHOS Rodent WB Antibody Cocktail ab110413; Abcam), anti-peptidylarginine deiminase type 4 (PAD4; 1:1,000; ab214810; Abcam), anti-TFAM (1:1,000; 18G102B2E11; Novus biologicals, Centennial, CO), anti-citrullinated histone H3 (1:1,000; ab5103; Abcam), and anti-MPO (1:1,000; ab9535; Abcam). The membranes were washed and incubated with secondary anti- mouse or rabbit IgG $(1: 2,000$; Kirkegaard \& Perry Laboratories, Inc., Gaithersburg, MD) at $25^{\circ} \mathrm{C}$ for $1 \mathrm{~h}$. The protein bands were detected using ImmunoStar ${ }^{\circledR}$ Zeta (Wako Pure Chemicals) and visualized using a LAS-4000 Mini imager (FUJIFILM, Tokyo, Japan).

PCR and reverse transcription PCR (RT-PCR). Neutrophillike HL-60 and $\rho^{0}$ cells were harvested by centrifugation of $1 \times 10^{6}$ cells per sample (and stored at $-30^{\circ} \mathrm{C}$ ). Nuclear DNA and mtDNA were isolated using the NucleoSpin ${ }^{\circledR}$ Tissue kit (TaKaRa Bio, Shiga, Japan). Total cellular DNA concentrations were assayed using a Nano-Drop spectrophotometer (Thermo Fisher Scientific). For RT-PCR, total RNA was isolated from all the cell groups by using ISOGEN II (NIPPON GENE, Tokyo, Japan). The isolated RNA was then reverse transcribed using a ReverTra Ace ${ }^{\circledR}$ kit (Toyobo, Osaka, Japan). The genomic DNA and 1st strand
cDNA were subjected to PCR with the following primer sets: Atp-6 (5'-atacacaacactaaaggacgaact-3', 5'-gaggcttactagaagtgaaaacg-3'), p47 $7^{\text {phox }} \quad\left(5^{\prime}\right.$-agtagcctgtgacgtcgtct-3', 5'-acccagccagcactatgtgt-3'), gp9 $1^{\text {phox }}\left(5^{\prime}\right.$-tctcctcatcatggtgcaca-3', 5'-gctgttcaatgcttgtggct-3'), p22 phox (5'-gtttgttttgtgcctgctggagt-3'，5'-tgggcggctgcttgatggt-3'), p67 $7^{\text {hox }}$ (5'-cgagggaaccagctgataga-3'，5'-catggaacactgagcttca-3'), cytochrome c oxidase 1 (5'-tccttattcgagccgagctg-3', 5'-gggctgtga cgataacgttg-3'), actin (5'-agagctacgagctgcctgac-3', 5'-agcactgtgttg gcgtacag-3'), and gapdh (5'-gagtccttccacgataccaaag-3', 5'-cccett cattgacctcaactac- $\left.3^{\prime}\right)$. The PCR products were loaded on $1 \%$ agarose gels and stained with ethidium bromide (EtBr).

Aminophenyl fluorescein (APF) assay. Neutrophil-like HL60 and $\rho^{0}$ cells were harvested by centrifugation of $1 \times 10^{6}$ cells and washed with PBS. The harvested cells were treated with $10 \mu \mathrm{M}$ APF just before $10 \mu \mathrm{M}$ A23187 or $10 \mathrm{nM}$ PMA stimulation. APF-stained cells were analyzed using flowcytometry $(488 \mathrm{~nm}$ excitation; $575 \mathrm{~nm}$ emission; BD FACS Caliber; BD Biosciences).

FACS analysis for CD11b. Cells $\left(1 \times 10^{6}\right)$ were incubated at $0^{\circ} \mathrm{C}$ for $30 \mathrm{~min}$ with the anti-CD $11 \mathrm{~b}$ antibody $(1: 100$; BD Biosciences), washed twice with PBS, and labeled with the FITCconjugated goat anti-mouse $\operatorname{IgG}$ (BD Biosciences) at $0^{\circ} \mathrm{C}$ for $30 \mathrm{~min}$. The cells were again washed with PBS and resuspended at $10^{6}$ cells $/ \mathrm{ml}$ in $2 \%$ formaldehyde in PBS. FACS analysis was performed using BD FACS Caliber.

Statistical analysis. Data are presented as mean \pm SD from at least three experiments. Statistical analysis was performed using Student's $t$ test or one way ANOVA and post hoc Tukey test.

\section{Results}

NET formation in mouse neutrophils from gp91 ${ }^{\text {phox }} \mathrm{KO}$ mice. Using gp9 $91^{\text {phox }} \mathrm{KO}$ mice, we analyzed the NOX-dependent and NOX-independent NET formation induced by PMA and A23187, respectively. On PMA stimulation, the gp91 ${ }^{\text {phox }} \mathrm{KO}$ mouse neutrophils did not produce NOX-derived ROS, whereas the wild-type mouse neutrophils generated large amounts of ROS (data not shown). Compared to the wild-type mouse neutrophils, the gp91 ${ }^{\text {phox }} \mathrm{KO}$ mouse neutrophils did not release DNA into the extracellular space after PMA stimulation (Fig. 1A and C). In contrast, A23187 stimulation did not induce ROS generation in gp91 ${ }^{\text {phox }} \mathrm{KO}$ mouse neutrophils (date not shown); however, there was no difference between the extracellular DNA release in gp9 ${ }^{\text {phox }} \mathrm{KO}$ and control mouse neutrophils (Fig. 1A and B). These results indicate that $\mathrm{A} 23187$ induced NET formation in gp91 $1^{\text {phox }}$ KO mice in a NOX-independent manner.

Effect of MitoTEMPO on NETs formation of neutrophillike HL-60 cells. ROS are produced not only by NOX, but also by mitochondria. Therefore, to evaluate the role of the mitochondrial ROS in the regulation of NET formation, we analyzed the effect of MitoTEMPO on NET formation in neutrophil-like HL-60 cells. MitoTEMPO is a mitochondria-targeted antioxidant that prevents mitochondrial oxidative damage. MitoTEMPO treatment slightly decreased the A23187- and PMA-induced NET formation (Fig. 2). Thus, MitoTEMPO did not completely suppress NET formation; therefore, we hypothesized that not only mitochondrial ROS but mitochondrial signaling is also involved in NET formation.

Establishment of $\rho^{0}$ cells from HL-60 cells. Further, to explore the involvement of mitochondria in NOX-dependent and -independent NET formation (Fig. 2), we generated $\rho^{0}$ cells from HL-60 cells. The previous method for generating $\rho^{0}$ cells involved a long-term culture (1-2 months) of cells in the presence of EtBr $(45 \mathrm{ng} / \mathrm{ml}) .{ }^{(14)}$ However, in this study, we used ddC, which prevents mtDNA replication, to establish $\rho^{0}$ cells in a short period ( 1 week). Mitochondrial deficiency of the resultant cells was confirmed by analyzing the expression of cytochrome c oxidase, which is encoded by the mtDNA (Fig. 3). Genomic PCR analysis and western blotting revealed that HL-60 cells treated with $1 \mu \mathrm{M}$ 


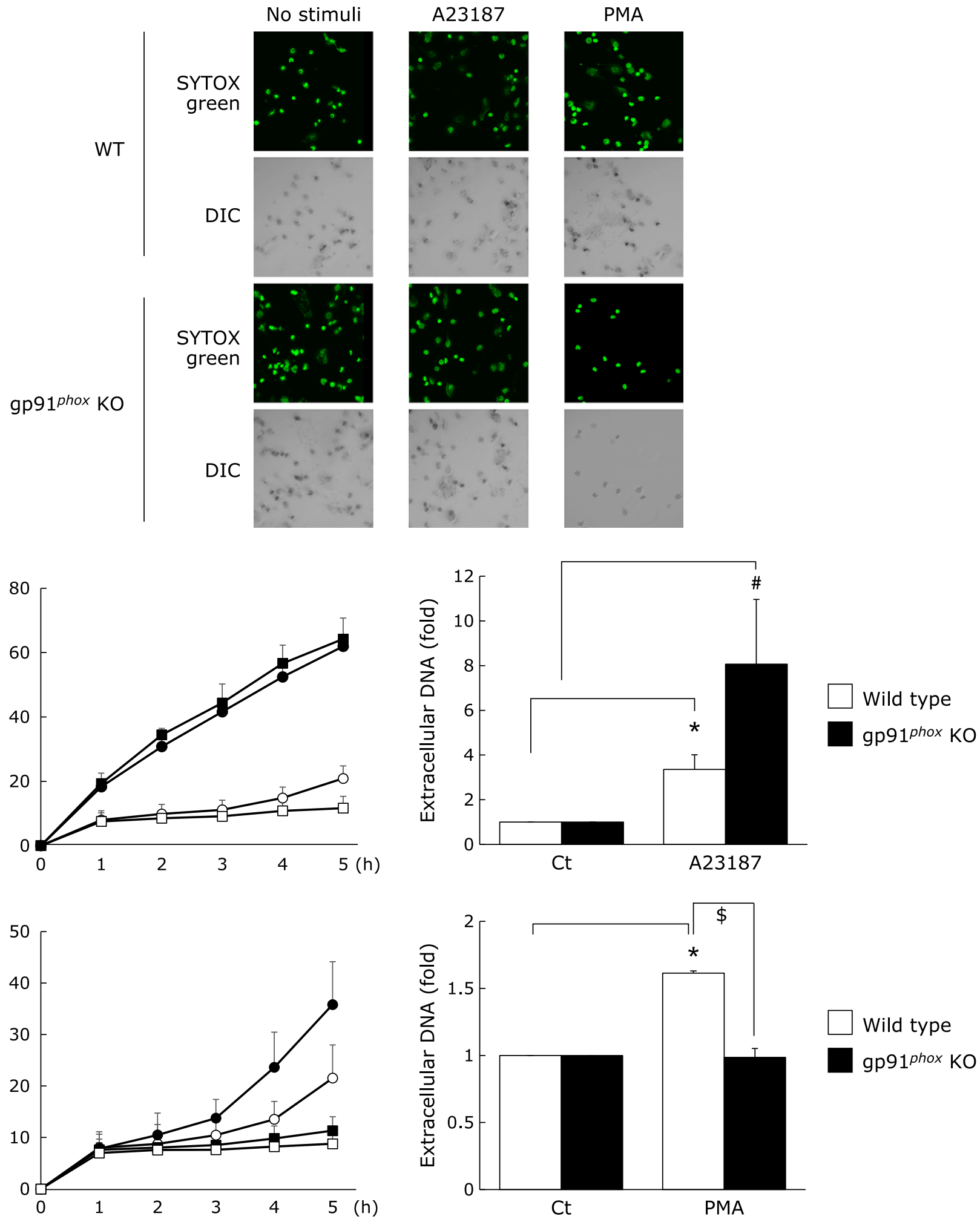

Fig. 1. Neutrophil extracellular trap (NET) formation in neutrophils from gp91 phox knockout (KO) mice and wild-type (WT) mice. The gp91 phox KO and WT mouse neutrophil were stimulated with $10 \mu \mathrm{M}$ A23187 (calcium ionophore) or $25 \mathrm{nM}$ or $1 \mu \mathrm{M}$ phorbol myristate acetate (PMA) for $3 \mathrm{~h}$, and stained SYTOX green $(5 \mu \mathrm{M})$, the cell-impermeable nucleic acid dye. (A) representative conforcal microscopy images showing NET formation; top panels: SYTOX green (DNA); bottom panels: differential interference contrast (DIC) images. (B) NETosis and extracellular DNA levels for A23187stimulated neutrophils. (C) NETosis and extracellular DNA levels for PMA-stimulated neutrophil. (O): stimulated WT mice, ( $\bigcirc)$ : unstimulated WT mice, ( $\square)$ : stimulated gp91 phox KO mice, $(\square)$ : unstimulated gp91 phox KO mice. Date represent mean \pm SD $(n=3)$. ${ }^{*} p<0.01$ A23187-treated cells (neutrophils from WT mice) vs Ct (unstimulated neutrophils from WT mice), ${ }^{*} p<0.05$ A23187-treated cells (neutrophils from gp91phox KO mice) vs $\mathrm{Ct}$ (unstimulated neutrophils from gp91 ${ }^{\text {phox }} \mathrm{KO}$ mice), ${ }^{5} p<0.01$ PMA-treated cells (neutrophils from gp91 ${ }^{\text {phox }} \mathrm{KO}$ mice) vs PMA-treated cells (neutrophils from WT mice). See color figure in the on-line version. 

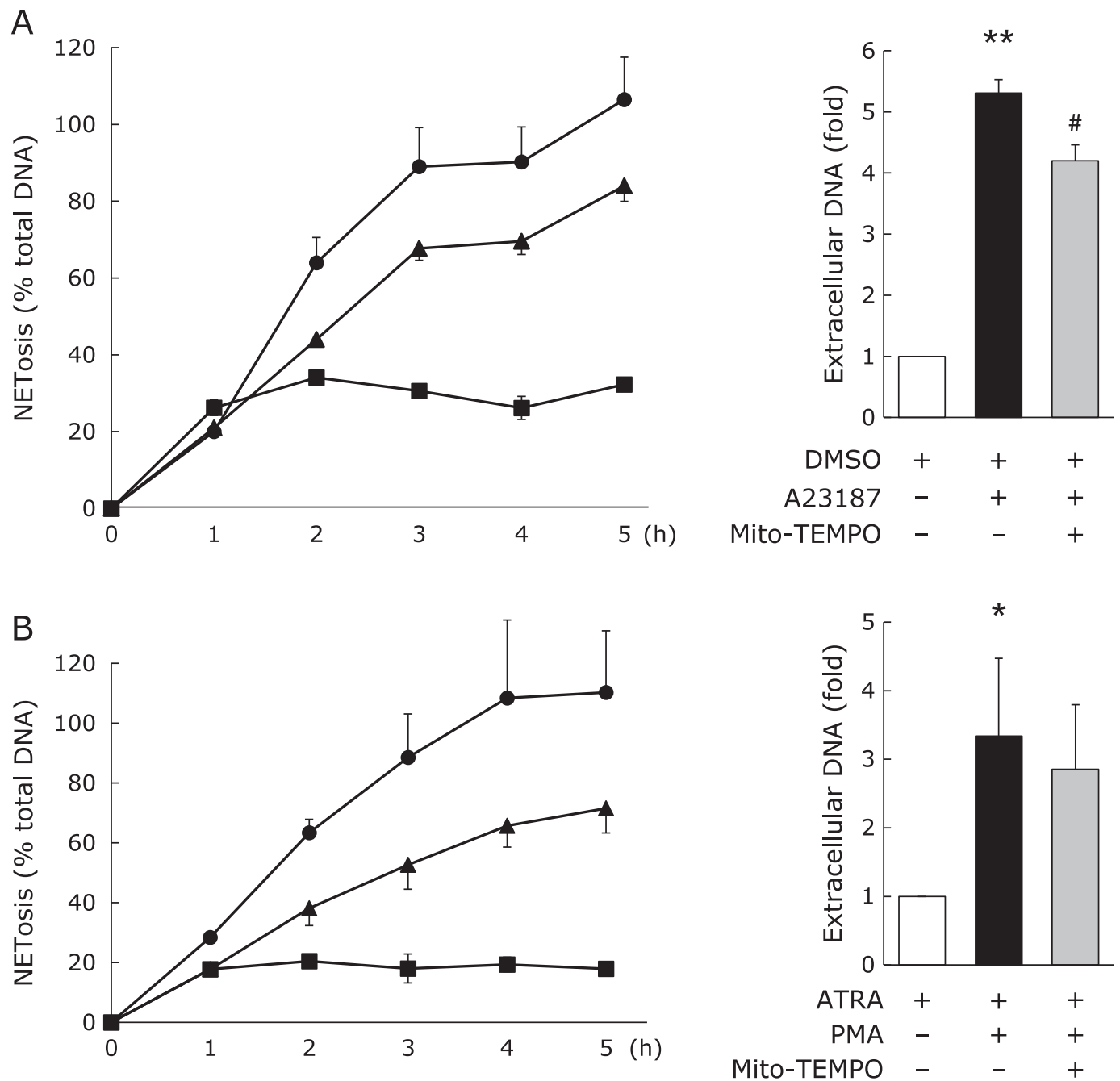

Fig. 2. Effect of MitoTEMPO on NET formation in neutrophil-like HL-60 cells. (A) NETosis and extracellular DNA levels for A23187-treated cells. $\mathrm{HL}-60$ cells were treated with $1.25 \%$ DMSO for $72 \mathrm{~h}$ for neutrophil differentiation. The resulting neutrophil-like cells were pretreated with $200 \mu \mathrm{M}$ MitoTEMPO for $30 \mathrm{~min}$ and then treated with $10 \mu \mathrm{M}$ A23187. (B) NETosis and extracellular DNA levels for phorbol myristate acetate (PMA)-treated cells. HL-60 cells were treated with $1 \mu \mathrm{M}$ ATRA for $72 \mathrm{~h}$ for neutrophil differentiation. The resulting neutrophil-like cells were pretreated with $200 \mu \mathrm{M}$ MitoTEMPO for $30 \mathrm{~min}$ and then treated with $25 \mathrm{nM}$ PMA. (O): stimulated cells, ( $\mathbf{\Delta}$ ): stimulated and MitoTEMPO-treated cells, ( $\mathbf{\square}$ ): unstimulated and untreated (control). ${ }^{*} p<0.05,{ }^{* *} p<0.01$ vs control, ${ }^{*} p<0.01$ vs A23187-stimulated cells.

A

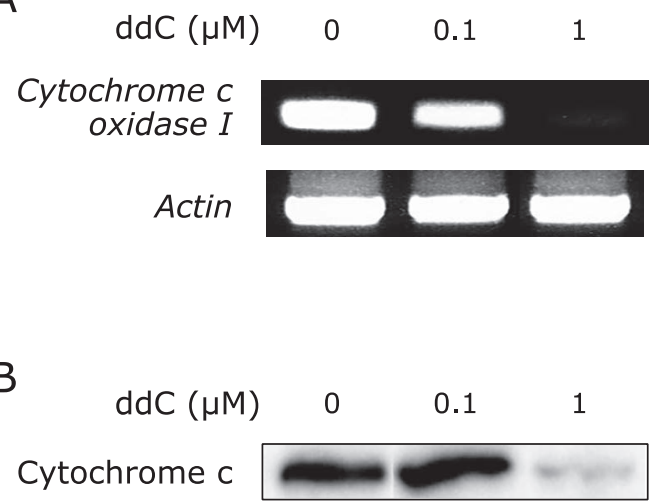

C

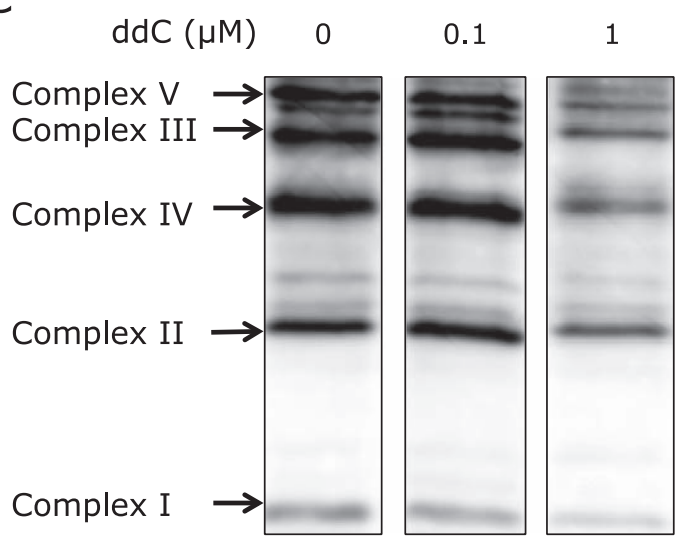

Fig. 3. Establishment of mitochondrial DNA deficient cells ( $\rho^{0}$ cells) from HL-60 cells. HL-60 cells were treated with or without 0.1 or $1 \mu \mathrm{M}$ ddC for 7 days. (A) Genomic PCR analysis showing the gene expression of cytochrome c oxidase 1 and actin. (B) Western blot showing the protein expression of cytochrome c. (C) Western blots showing the protein expression of mitochondrial complexes. 
A

0 day

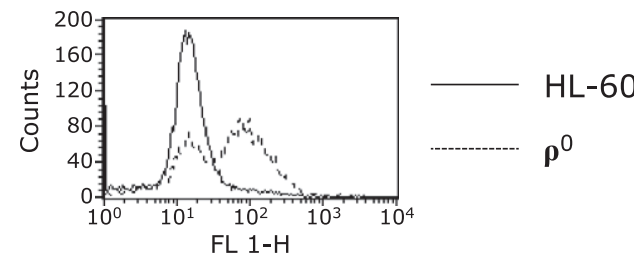

1 day
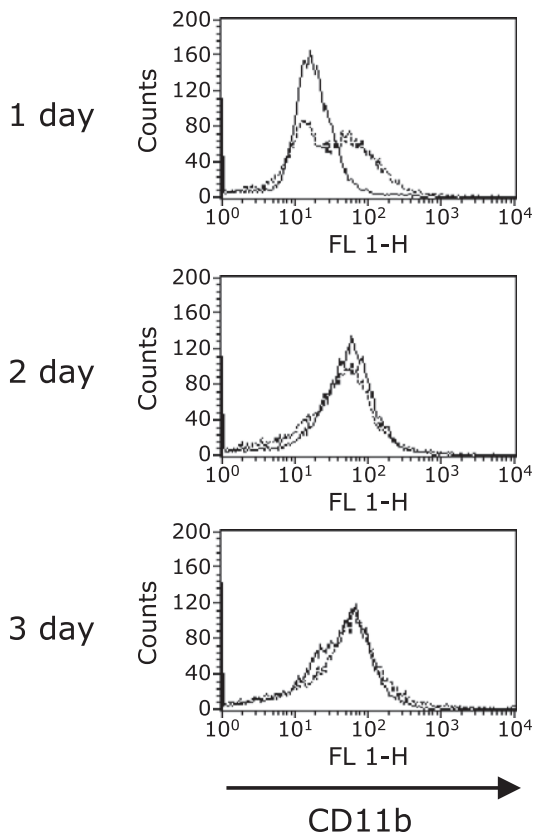

C

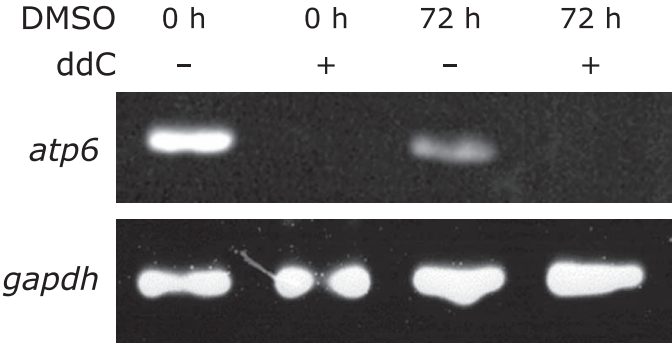

B

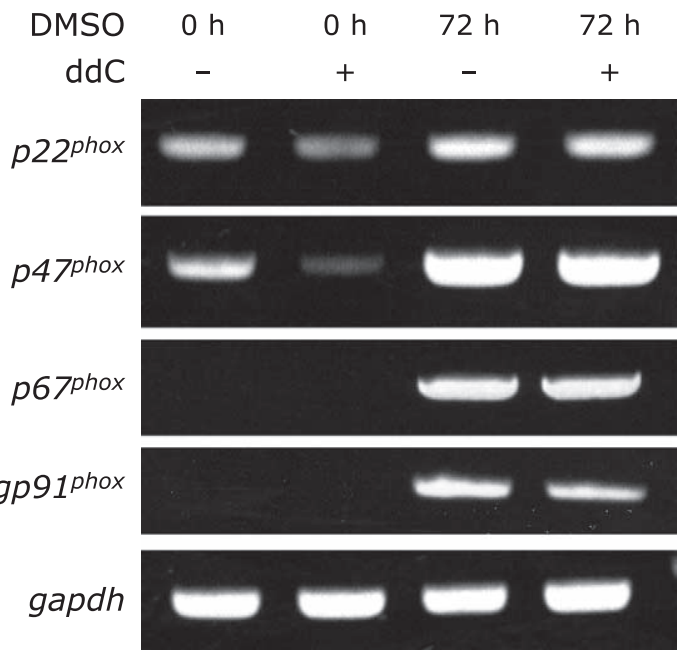

$\mathrm{D}$
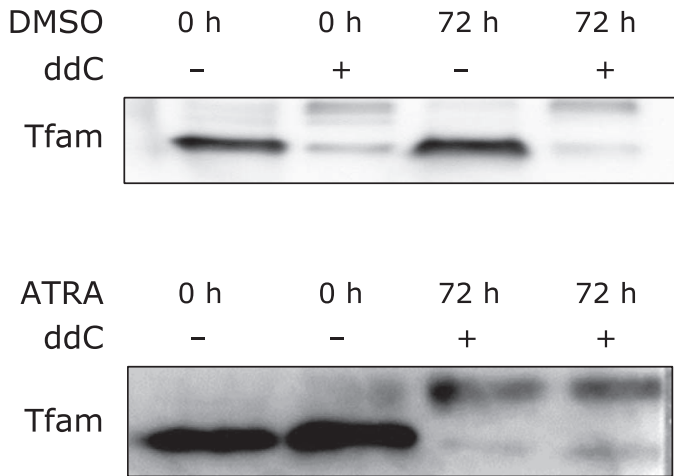

Fig. 4. Characterization of neutrophil-like $\rho^{0}$ cells. HL-60 and $\rho^{0}$ cells were treated with $1.25 \%$ DMSO for 3 days. (A) Flow cytometry results showing CD $11 \mathrm{~b}$ expression from 0 day to 3 days after DMSO treatment. (B) RT-PCR results showing the expression of NADPH oxidase complex coponents

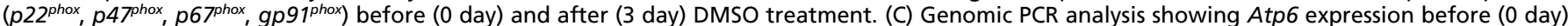
and after (3 day) DMSO treatment. (D) Western blot showing TFAM expression before (0 day) and after (3 day) DMSO treatment.

ddC for 7 days depleted gene and protein expression of cytochrome C (Fig. 3A and B). Moreover, the protein expression of mitochondrial complexes I-V was also markedly decreased in the ddC treated HL-60 cells (Fig. 1C). Thus, we established $\rho^{0}$ cells from HL-60 cells using ddC for 1 week.

Neutrophil-differentiation of $\rho^{0}$ cells. To confirm the effect of mtDNA depletion on neutrophil differentiation, we treated HL-60 cells and $\rho^{0}$ cells with $1.25 \%$ DMSO for neutrophil induction. We analyzed the expression of CD11b, a neutrophil surface antigen, using flow cytometry. CD11b was expressed on day 3 of differentiation in both HL- 60 and $\rho^{0}$ cells (Fig. 4A). There was no difference in the mRNA expression of NADPH oxidase complex components (gp91 phox $, \mathrm{p} 22^{\text {phox }}, \mathrm{p} 47^{\text {phox }}$, and p67 $7^{\text {phox }}$ ) of HL-60 and $\rho^{0}$ cells on day 3 (Fig. 4B). Atp6, which is encoded in mtDNA, was markedly decreased in the DMSO-induced neutrophil-like $\rho^{0}$ cells (Fig. 4C). The expression of TFAM, a transcription factor of mitochondria, also decreased in $\rho^{0}$ cells and neutrophil-like $\rho^{0}$ cells (Fig. 4D). these findings confirm that $\rho^{0}$ cells remain mtDNA deficient even after differentiation and that there was no difference in neutrophil differentiation between HL- 60 and $\rho^{0}$ cells.

NET formation in neutrophil-like HL-60 and $\rho^{0}$ cells.

Next, to elucidate the role of mitochondria in NET formation and extracellular DNA release in neutrophil-like $\rho^{0}$ cells. After A23187 stimulation, extracellular DNA release from neutrophillike $\rho^{0}$ cells was significantly lower than that from neutrophil-like HL-60 cells (Fig. 5A). However, there was no difference between the PMA-induced extracellular DNA release in neutrophil-like HL-60 cells and neutrophil-like $\rho^{0}$ cells (Fig. 5B). These results suggest that mitochondrial function is essential for NOX-independent NET formation.

Citrullination of histone $\mathrm{H3}$ in neutrophil-like HL-60 and $\rho^{0}$ cells. Histone H3 citrullination plays a critical role in NET formation. Therefore, we analyzed H3 citrullination in neutrophillike HL-60 and $\rho^{0}$ cells using western blotting. Compared to the 
A
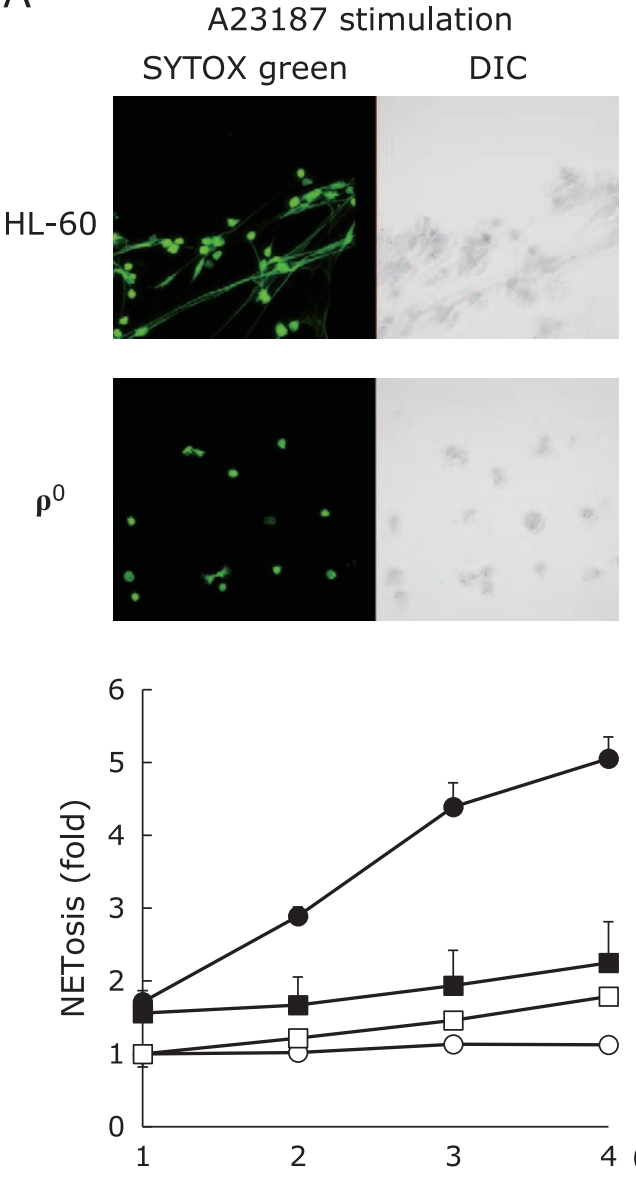

(h)

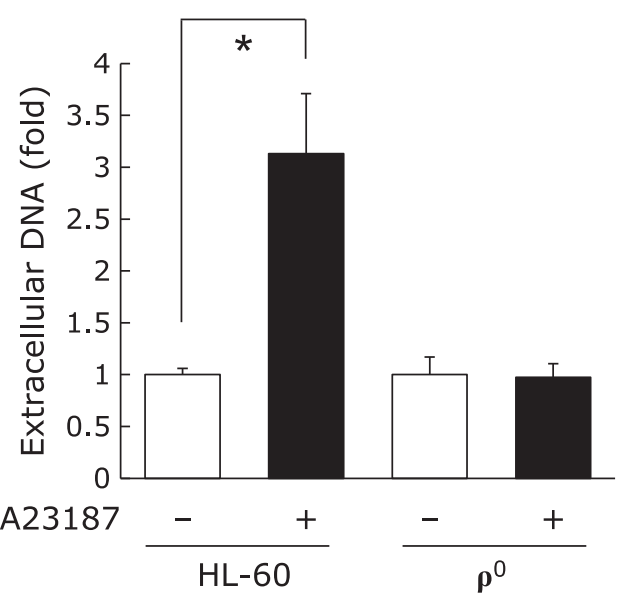

B
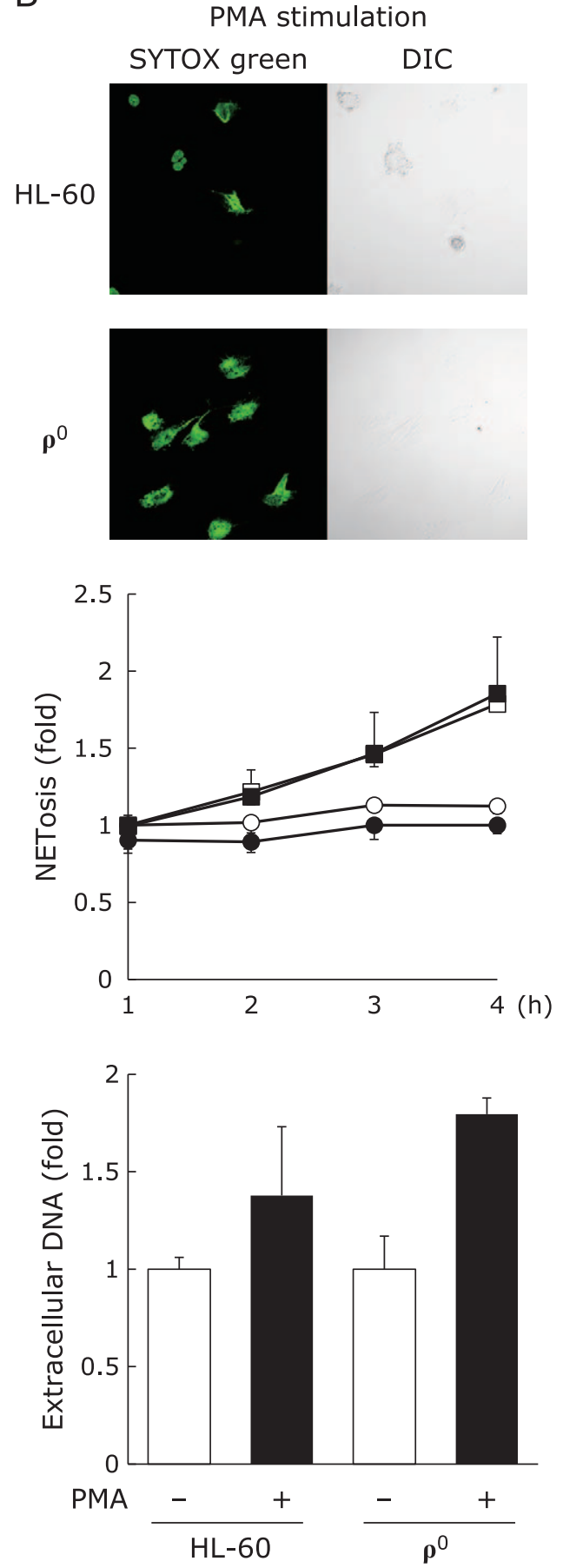

Fig. 5. Analysis of NET formation of $\rho^{0}$ cells in neutrophil-like HL-60 and $\rho^{0}$ cells. HL-60 and $\rho^{0}$ cells were treated with $1.25 \%$ DMSO for $72 \mathrm{~h}$, and then treated with (A) $10 \mu \mathrm{M}$ A23187 or (B) $10 \mathrm{nM}$ PMA for $4 \mathrm{~h}$. Representative confocal microscopy images showing NETosis have been provided; top left panels:SYTOX green (DNA); top right panels: differential interference contrast (DIC) images. The middle and bottom panels show NETosis and extracellular DNA levels. (O): stimulated cells, $(\mathbf{\square})$ : unstimulated cells, $(\square)$ : stimulated $\rho^{0}$ cells, $(\bigcirc)$ : unstimulated $\rho^{0}$ cells. ${ }^{*} p<0.05$ vs control. See color figure in the on-line version.

controls, A23187-stimulated neutrophil-like HL-60 and $\rho^{0}$ cells showed increased expression of PAD4, an enzyme that converts histone arginine residues to citrulline (Fig. 6A), and increased expression of citrullinated H3 (Fig. 6B). Thus, although A23187 stimulation did not induce NET formation in neutrophil-like $\rho^{0}$ cells, H3 citrullination was induced by A23187 stimulation. These results suggest that inhibition of NOX-independent NET formation did not affect PAD4 expression and histone citrullination.
Cell membrane disruption during NET formation in neutrophil-like HL-60 cells. As H3 citrullination was not affected by mitochondrial deficiency, we next, investigated the cell membrane disruption mechanism in neutrophil-like HL-60 cells. $\mathrm{HClO}^{-}$production by MPO was reported to be important for membrane disruption during NET formation. ${ }^{(15,16)}$ Therefore, we investigated the effect of ABAH, an MPO inhibitor, on NET formation. ABAH treatment did not suppress A23187-induced 
A

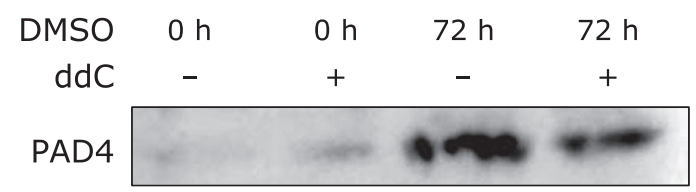

B

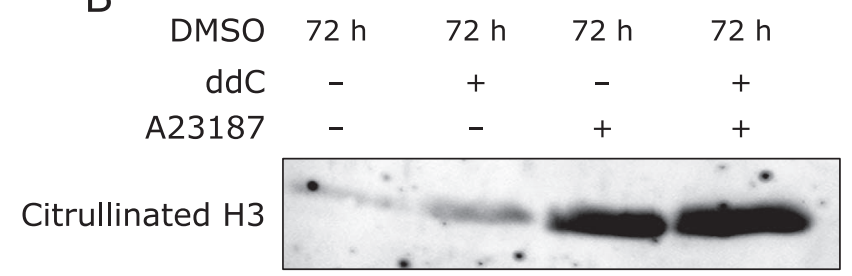

Fig. 6. Peptidylarginine deiminase type 4 (PAD4) expression and histone $\mathrm{H} 3$ citrullination in neutrophil-like $\mathrm{HL}-60$ and $\rho^{0}$ cells. Neutrophillike HL-60 and $\rho^{0}$ cells were treated with $10 \mu \mathrm{M}$ A23187 and nuclear extraction was performed. Western blotting was performed to analyze the expression of (A) PAD4 and (B) citrullination H3 (normalized to H3 protein levels).

NET formation (Fig. 7A) but markedly suppressed PMA-induced NET formation (Fig. 7B). Therefore, we measured $\mathrm{HClO}^{-}$generation using APF staining in neutrophil-like HL-60 and $\rho^{0}$ cells. $\mathrm{HClO}^{-}$generation was observed in both neutrophil-like HL60 and $\rho^{0}$ cells after PMA stimulation but not after A23187 stimulation (Fig. 7C).

Therefore, to further investigate the other potential mechanism of membrane disruption in A23187- and PMA-induced NET formation, we investigated the effect of NSA, an MLKL inhibitor on NET formation in neutrophil-like HL-60 cells. NSA treatment suppressed both A23187- and PMA-induced NET formation (Fig. 7D and E). These data suggest the existence of different necroptosis mechanisms in NOX-dependent and NOX-independent NET formation.

\section{Discussion}

In this study, we investigated NOX-dependent and -independent NET formation in mtDNA-deficient cells ( $\rho^{0}$ cells). We showed that mitochondria play an important role in the NOX-independent NET formation. Furthermore, membrane disruption in NOXdependent NET formation occurred via MPO and MLKL, whereas that in NOX-independent NET formation was MPO-independent and was partially induced in an MLKL-dependent manner.

It is known that NET formation is induced in a NOX-dependent manner. ${ }^{(1)}$ Neutrophils from gp9 $1^{\text {phox }} \mathrm{KO}$ mice do not show NET formation in response to NOX-activating stimulants. ${ }^{(17)}$ In this study, the neutrophils from gp91 $1^{\text {phox }} \mathrm{KO}$ mice showed NET formation after A23187 stimulation but not after PMA stimulation. This indicates that NET formation occurs via NOXindependent mechanisms in gp $91^{\text {phox }} \mathrm{KO}$ mice. Moreover, inhibition of mitochondrial ROS production decreased NET formation induced via both NOX-dependent and -independent mechanisms (Fig. 2) suggesting that both the mechanisms involve mitochondrial ROS generation.

Previous studies demonstrated mtDNA deletion using EtBr. ${ }^{(14)}$ However, this method requires long-term culture ( 2 months) with $\mathrm{EtBr}$, and the agent can potentially affect the genomic DNA of the cells. Therefore, in this study, we generated $\rho^{0}$ cells from HL-60 cells using a novel method involving $\mathrm{ddC}^{(18)}$ This innovative method required a short-term treatment ( 7 days) and provides a more efficient and highly reproducible alternative to generate $\rho^{0}$ cells from HL-60 cells. Furthermore, the expression of CD11b, a differentiation marker, occurred earlier in $\rho^{0}$ cells than in HL-60 cells (Fig. 4A), indicating that $\rho^{0}$ cells might differentiate more rapidly than HL-60 cells.

NOX-independent NET formation after NADPH oxidase inhibition has been reported in human peripheral neutrophils. ${ }^{(10)}$ However, the mechanism of NOX-independent NET formation remains unclear. A recent study involving SK3 and mitochondrial ROS inhibitor suggested that calcium-activated NOX-independent NET formation is fast and mediated by SK3 and mitochondrial ROS. ${ }^{(10)}$ In this study, we investigated the role of mitochondrial pathway by using $\rho^{0}$ cells, which lack the mitochondrial ROS generation and signal transduction..$^{(9,19)}$ Our results were consistent with the previous results obtained using pharmacological approach, and confirm that mitochondrial signaling is essential for NOXindependent NET formation. Our findings also suggest that mitochondria do not affect NOX-dependent NET formation.

Several studies have reported the relation between MPO and NET formation. ${ }^{(20-22)}$ In particular, NET formation in MPOdeficient neutrophils was reported to be induced by calcium ionophore (ionomycin) but not by PMA. ${ }^{(23)}$ Furthermore, it was suggested that cell membrane destruction depends on MPO. Therefore, to clarify the involvement of MPO in cell membrane disruption mechanism, we analyzed the effects of pharmacological inhibition of MPO in this study. In neutrophil-like HL-60 cells, treatement with the MPO inhibitor ABAH significantly suppressed PMA-induced NET formation, but not A23187-induced NET formation (Fig. 7A and B). Interestingly, in both HL-60 and $\rho^{0}$ cells, H3 citrullination occurred without membrane disruption (Fig. 6). Thus, membrane disruption occurred via different pathways during NOX-dependent and -independent NET formation. In NOX-dependent NET formation, cell membrane disruption occurred because of $\mathrm{HClO}^{-}$generated by MPO. ${ }^{(24)}$ However, the involvement of MPO-generated $\mathrm{HClO}^{-}$was not observed in NOX-independent NET formation; thus, the mechanism of cell membrane disruption in NOX-independent NET formation remained unclear. Recently, it was reported that anti-neutrophil cytoplasmic antibody (ANCA) induced NET formation via receptor-interacting protein kinase (RIPK) 1/3- and MLKLdependent necroptosis. ${ }^{(25,26)}$ Another report also demonstrated that the RIPK-1 stabilizers necrostatin-1 or necrostatin-1s and the MLKL inhibitor NSA prevent monosodium urate crystal- or PMA-induced NET formation in human and mouse neutrophils. ${ }^{(27)}$ Therefore, we examined the effect of the NSA on NOX-dependent and -independent NET formation (Fig. 7). NSA partially inhibited both NOX-dependent and NOX-independent NET formation. Thus, membrane disruption in NOX-dependent NET formation involves both MPO-mediated $\mathrm{HClO}^{-}$generation and MLKL activation, whereas membrane disruption in NOX-independent NET formation partially occurs via MLKL activity.

In conclusion, we generated mtDNA-deficient cells and showed that mitochondria, but not mitochondrial ROS, affected NOXindependent NET formation. Moreover, we show that cell membrane disruption in NOX-dependent NET formation occurs via both MPO- and MLKL-dependent mechanisms and that cell membrane disruption in NOX-independent NET formation partially occurs via an MLKL-dependent mechanism.

\section{Abbreviations}

ABAH 4-aminobenzoic acid hydrazide

APF aminophenyl fluorescein

ATRA all-trans retinoic acid

ddC dideoxycytidine

DMSO dimethyl sulfoxide

$\mathrm{EtBr}$ ethidium bromide

KO knockout

MAPK mitogen-activated protein kinase 

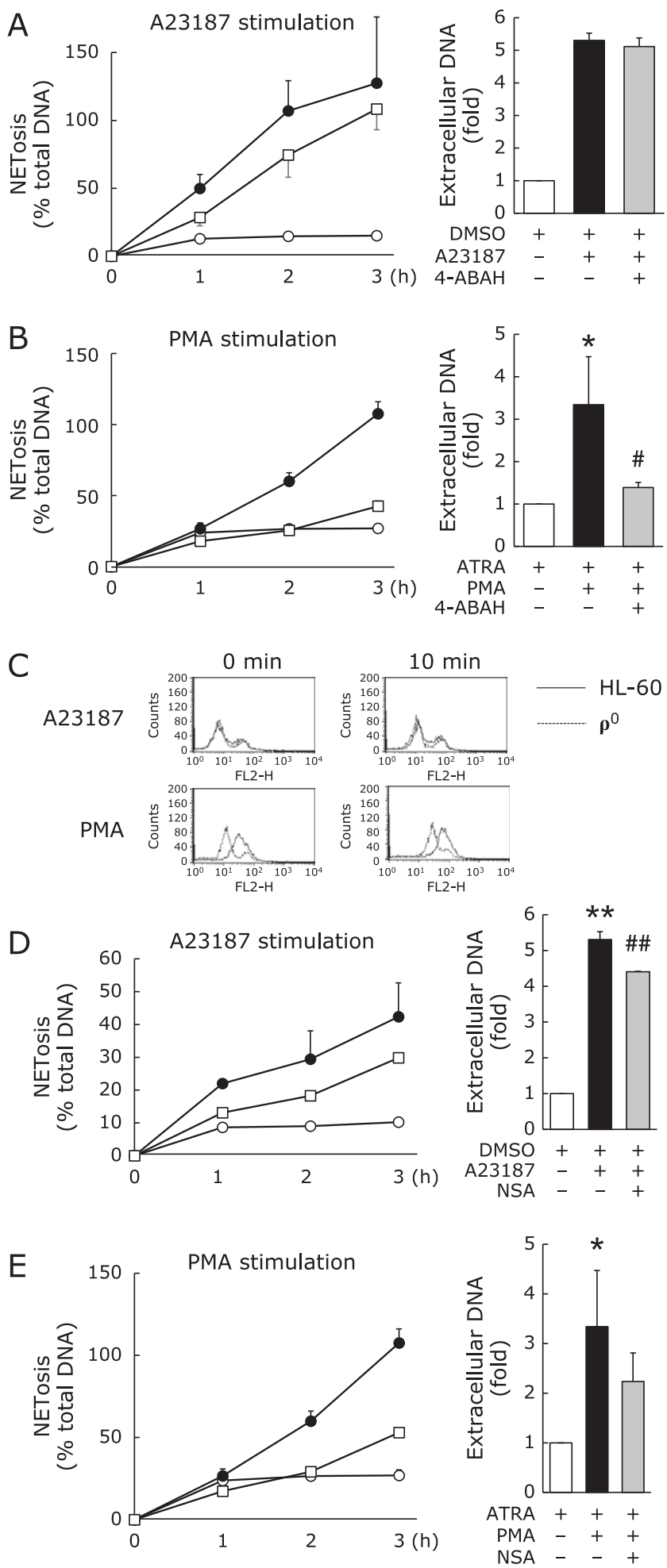

Fig. 7. Analysis of plasma membrane disruption during NET formation in neutrophil-like HL-60 cells. (A) NETosis and extracellular DNA levels in A23187-stimulated HL-60 cells with or without ABAH treatment. HL-60 cells were treated with $1.25 \%$ DMSO for $72 \mathrm{~h}$ for neutrophil differentiation. The resulting neutrophil-like cells were treated with $500 \mu \mathrm{M}$ ABAH for $3 \mathrm{~h}$ and then with $10 \mu \mathrm{M}$ A23187. (B) NETosis and extracellular DNA levels for phorbol myristate acetate (PMA)-treated HL-60 cells with or without ABAH treatment. HL-60 cells were treated with $1 \mu \mathrm{M}$ ATRA for $72 \mathrm{~h}$ for neutrophil differentiation. The resulting neutrophil-like cells were treated with $500 \mu \mathrm{M}$ ABAH for $3 \mathrm{~h}$ and then with $25 \mathrm{nM}$ PMA. (C) Aminophenyl Fluorescein (APF)-based flow cytometry analysis of $\mathrm{HClO}$ - generation before $(0 \mathrm{~min})$ and at $10 \mathrm{~min}$ after A23187 or PMA stimulation. (D) NETosis and extracellular DNA levels in A23187-stimulated HL-60 cells with or without necrosulfonamide (NSA) treatment. HL-60 cells were treated with $1.25 \%$ DMSO for $72 \mathrm{~h}$ for neutrophil differentiation. The resulting neutrophil-like cells were treated with $50 \mu \mathrm{M}$ NSA for $30 \mathrm{~min}$ and then with $10 \mu \mathrm{M}$ A23187. (E) NETosis and extracellular DNA levels for phorbol myristate acetate (PMA)-treated HL-60 cells with or without NSA treatment. HL-60 cells were treated with $1 \mu \mathrm{M}$ ATRA for $72 \mathrm{~h}$ for neutrophil differentiation. The resulting neutrophil-like cells were treated with $50 \mu \mathrm{M}$ NSA for 30 min and then with $25 \mathrm{nM}$ PMA. (O): stimulated cells, $(\square)$ : stimulated and inhibitor-treated cells, $(\bigcirc)$ : unstimulated and untreated (control). ${ }^{*} p<0.05$, ${ }^{* *} p<0.01$ vs control, ${ }^{*} p<0.01,{ }^{\# \#} p<0.01$ vs A23187-stimulated cells. 
MLKL

MPO

mixed lineage kinase domain like pseudokinase

myeloperoxidase

mtDNA mitochondrial DNA

NSA necrosulfonamide

NET neutrophil extracellular trap

NETosis NET-associated cell death

NOX NADPH oxidase

PAD4 peptidyl arginine deiminase 4

PMA phorbol myristate acetate

\section{References}

1 Fuchs TA, Abed U, Goosmann C, et al. Novel cell death program leads to neutrophil extracellular traps. J Cell Biol 2007; 176: 231-241.

2 Porto BN, Stein RT. Neutrophil extracellular traps in pulmonary diseases: too much of a good thing? Front Immunol 2016; 7: 311.

3 Bicker KL, Thompson PR. The protein arginine deiminases: structure, function, inhibition, and disease. Biopolymers 2013; 99: 155-163.

4 Hakkim A, Fürnrohr BG, Amann K, et al. Impairment of neutrophil extracellular trap degradation is associated with lupus nephritis. Proc Natl Acad Sci U S A 2010; 107: 9813-9818.

5 Fuchs TA, Brill A, Wagner DD. Neutrophil extracellular trap (NET) impact on deep vein thrombosis. Aeterioscler Thromb Vasc Biol 2012; 32: 17771783.

6 Arai Y, Nishinaka Y, Arai T, et al. Uric acid induces NADPH oxidaseindependent neutrophil extracellular trap formation. Biochem Biophys Res Commun 2014; 443: 556-561.

7 Bianchi M, Hakkim A, Brinkmann V, et al. Restoration of NET formation by gene therapy in CGD controls aspergillosis. Blood 2009; 114: 2619-2622.

8 Konig MF, Andrade F. A critical reappraisal of neutrophil extracellular traps and NETosis mimics based on differential requirements for protein citrullination. Front Immunol 2016; 7: 461.

9 Kenny EF, Herzig A, Krüger R, et al. Diverse stimuli engage different neutrophil extracellular trap pathways. ELife 2017; 6. pii: e24437.

10 Douda DN, Khan MA, Grasemann H, Palaniyar N. SK3 channel and mitochondrial ROS mediate NADPH oxidase-independent NETosis induced by calcium influx. Proc Natl Acad Sci U S A 2015; 112: 2817-2822.

11 Kasahara E, Sekiyama A, Hori M, et al. Mitochondrial density contributes to the immune response of macrophages to lipopolysaccharide via the MAPK pathway. FEBS Lett 2011; 585: 2263-2268.

12 Hara K, Kasahara E, Takahashi N, et al. Mitochondria determine the efficacy of anticancer agents that interact with DNA but not the cytoskeleton. $J$ Pharmacol Exp Ther 2011; 337: 838-845.

13 Manda-Handzlik A, Bystrzycka W, Wachowska M, et al. The influence of agents differentiating HL-60 cells toward granulocyte-like cells on their ability to release neutrophil extracellular traps. Immunol Cell Biol 2018; 96: 413-425.

14 Vaux EC, Metzen E, Yeates KM, Ratcliffe PJ. Regulation of hypoxiainducible factor is preserved in the absence of a functioning mitochondrial respiratory chain. Blood 2001; 98: 296-302.

15 Chen MS, Lin WC, Yeh HT, Hu CL, Sheu SM. Propofol specifically reduces PMA-induced neutrophil extracellular trap formation through inhibition of p-ERK and HOCl. Life Sci 2019; 221: 178-186.
ROS reactive oxygen species

SK3 small conductance calcium-activated potassium channel protein 3

$\rho^{0}$ cells mitochondrial DNA-deficient cells

\section{Conflict of Interest}

No potential conflicts of interest were disclosed.

16 Parker H, Albrett AM, Kettle AJ, Winterbourn CC. Myeloperoxidase associated with neutrophil extracellular traps is active and mediates bacterial killing in the presence of hydrogen peroxide. J Leukoc Biol 2012; 91: 369376.

17 Ermert D, Urban CF, Laube B, Goosmann C, Zychlinsky A, Brinkmann V. Mouse neutrophil extracellular traps in microbial infections. J Innate Immun 2009; 1: 181-193.

18 Brown TA, Clayton DA. Release of replication termination controls mitochondrial DNA copy number after depletion with 2',3'-dideoxycytidine. Nucleic Acids Res 2002; 30: 2004-2010.

19 Kimoto-Kinoshita S, Nishida S, Tomura TT. Diethyldithiocarbamate can induce two different type of death: apoptosis and necrosis mediating the differential MAP kinase activation and redox regulation in HL60 cells. Mol Cell Biochem 2004; 265: 123-132.

20 Parker H, Dragunow M, Hampton MB, Kettle AJ, Winterbourn CC Requirements for NADPH oxidase and myeloperoxidase in neutrophil extracellular trap formation differ depending on the stimulus. J Leukoc Biol 2012; 92: 841-849.

21 Yoshida M, Yamada M, Sudo Y, et al. Myeloperoxidase anti-neutrophil cytoplasmic antibody affinity is associated with the formation of neutrophil extracellular traps in the kidney and vasculitis activity in myeloperoxidase anti-neutrophil cytoplasmic antibody-associated microscopic polyangiitis. Nephrology (Carlton) 2016; 21: 624-629.

22 Muñoz-Caro T, Lendner M, Daugschies A, Hermosilla C, Taubert A NADPH oxidase, MPO, NE, ERK1/2, p38 MAPK and $\mathrm{Ca}^{2+}$ influx are essential for Cryptosporidium parvum-induced NET formation. Dev Comp Immunol 2015; 52: 245-254.

23 Parker H, Winterbourn CC. Reactive oxidants and myeloperoxidase and their involvement in neutrophil extracellular traps. Front Immunol 2013; 3: 424.

24 Winterbourn CC, Kettle AJ, Hampton MB. Reactive oxygen species and neutrophil function. Annu Rev Biochem 2016; 85: 765-792.

25 Amini P, Stojkov D, Wang X, et al. NET formation can occur independently of RIPK3 and MLKL signaling. Eur J Immunol 2016; 46: 178-184.

26 Schreiber A, Rousselle A, Becker JU, von Mässenhausen A, Linkermann A, Kettritz R. Necroptosis controls NET generation and mediates complement activation, endothelial damage, and autoimmune vasculitis. Proc Natl Acad Sci U S A 2017; 114: E9618-E9625.

27 Desai J, Kumar SV, Mulay SR, et al. PMA and crystal-induced neutrophil extracellular trap formation involves RIPK1-RIPK3-MLKL signaling. Eur $J$ Immunol 2016; 46: 223-229. 